\title{
Two-Stage Least Squares based Iterative Identification Algorithm for Box-Jenkins Model
}

\author{
Jie Jia ${ }^{1}$, Hua Huang ${ }^{1}$, Yong Yang ${ }^{2, *}$, Ke Lv ${ }^{3}$ and Feng Ding ${ }^{4}$ \\ ${ }^{1}$ Institute of Aerospace Information and Security Technology, Nanchang Hangkong University, Nanchang 330063, China \\ ${ }^{2}$ School of Information Technology, Jiangxi University of Finance and Economics, Nanchang 330032, China \\ ${ }^{3}$ School of Computing and Communication Engineering, University of Chinese Academy of Sciences, Beijing 100049, China \\ ${ }^{4}$ School of Internet of Things Engineering, Jiangnan University, Wuxi 214122, China
}

Received: 23 Jun. 2013, Revised: 28 Oct. 2013, Accepted: 29 Oct. 2013

Published online: 1 May. 2014

\begin{abstract}
In order to improve the parameters estimation precision, a two-stage least squares iterative algorithm for Box-Jenkins models is presented, which is based on the interactive estimation theory of the hierarchical identification and the auxiliary model. The main idea of the algorithm is to decompose a Box-Jenkins system into two subsystems so as to identify each subsystem, respectively. In our algorithm, the dimensions of the involved covariance matrices in each subsystem turn to be small. The simulation results indicate that the proposed algorithm is effective and has a high computational efficiency.
\end{abstract}

Keywords: Box-Jenkins model, parameter estimation, hierarchical identification, covariance

\section{Introduction}

Actual system often has interference effect, and the interference is often with colored noise. Under the colored noise interference, many identification methods are used to estimate parameters for Box-Jenkins model [1,2,3,4,5]. In order to get unbiased estimation parameters of the model, the recursive extended least squares algorithm (RELS) for CARMA model was extended to Box-Jenkins model, and then the recursive generalized extended least squares algorithm (RGELS) was presented [6]. The structure and parameter estimation of Box-Jenkins model were obtained by using UD decomposition [7]. The deviation compensation least-squares identification method and its recursive form were pushed by using deviation compensation principle [8,9]. The drawback of deviation compensation least squares identification method is that the parameters of noise model are not identified. But the recursive extended least-squares algorithm is simple and easily to realized, not only can identify the parameters of the system model, but also parameter estimation of the noise model can be obtained [10,11].

Least-squares iteration identification method use all the data of the system in the process of every step iterative calculation, and extract information as much as possible from the data to improve the parameter estimation accuracy[12,13,14].For example, Ding et al. presented least squares based iterative algorithms for Hammerstein nonlinear ARMAX systems[14], and for OE and OEMA systems[15]; Han et al. gave a hierarchical least squares based iterative identification algorithm for a class of multivariable CARMA-like systems[16]; Zhang et al. Proposed a hierarchical gradient based iterative parameter estimation algorithm for multi-variable output error Moving average systems [17]; Bao et al. developed a least squares based iterative identification method for multi-variable controlled ARMA systems[18]; Ding et al. Presented a least squares based iterative algorithm for controlled auto-regressive auto-regressive moving average (CARARMA) systems[19]. Also, a least squares based iterative algorithm is developed for BoxCJenkins systems, and the precision of parameter estimation is improved compared with recursive generalized extended least squares algorithm[20].

However, because the dimensions of the involved covariance matrices are big, lead to large amount of calculation[20]. The idea of decomposition technique and the auxiliary model identification were adopted in this paper, and then the two-stage [21,22, 23, 24] least-squares iteration identification method was proposed for Box-

\footnotetext{
*Corresponding author e-mail: yangyong5080@126.com
} 
Jenkins model. The basic idea is to decompose a BoxJenkins system into two subsystems and to identify each subsystem, respectively. Because the dimensions of the involved covariance matrices in each subsystem become small, the proposed algorithm has a high computational efficiency[25].

\section{Model description}

Let us introduce some notations first. " $\mathrm{A}:=\mathrm{X}$ " or " $\mathrm{X}:=\mathrm{A}$ " stands for "A is defined as X"; the symbol $I$ stands for an identity matrix of appropriate size $(n \times n)$; the superscript $T$ denotes the matrix transpose; $I_{n}$ represents an n-dimensional column vector whose elements are all 1.

Consider the Box-Jenkins system, depicted in Fig.1,

$$
y(t)=\frac{B(z)}{A(z)} u(t)+\frac{D(z)}{C(z)} v(t)
$$

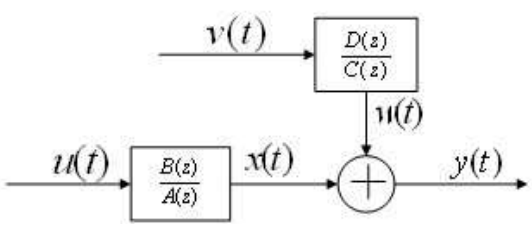

Fig. 1 A system described byt he Box-Jenkins model

Where, $y(t)$ and $u(t)$ are the input and output sequences of the system, respectively, $v(t)$ is a white noise sequence with zero mean and variance $\sigma^{2}$, and $A(z), B(z)$, $C(z)$ and $D(z)$ are the polynomials in the unit backward shift operator $Z^{-1}\left(\left[Z^{-1} y(t)=y(t-1)\right]\right)$, and defined by,

$$
\begin{aligned}
& A(z):=1+a_{1} z^{-1}+a_{2} z^{-2}+\cdots+a_{n_{a}} z^{-n_{a}} \\
& B(z):=b_{1} z^{-1}+b_{2} z^{-2}+\cdots+b_{n_{b}} z^{-n_{b}} \\
& C(z):=1+c_{1} z^{-1}+c_{2} z^{-2}+\cdots+c_{n_{c}} z^{-n_{c}} \\
& D(z):=1+d_{1} z^{-1}+d_{2} z^{-2}+\cdots+d_{n_{d}} z^{-n_{d}}
\end{aligned}
$$

Without loss of generality, assume that $u(t)=0, y(t)=$ $0, v(t)=0$ for $t \leq 0$, and known orders $\left(n_{a}, n_{b}, n_{c}, n_{d}\right)$. The objective of this paper is to apply the decomposition technique and derive a two-stage least squares based iterative identification algorithm for estimating the system parameters, and decompose the original identification system into two subsystems with smaller order problem.

Define the system parameter vectors and the information vectors,

$$
\begin{gathered}
\theta:=\left[\begin{array}{c}
\theta_{s} \\
\theta_{n}
\end{array}\right] \in R^{n} \\
\theta_{s}:=\left[a_{1}, a_{2}, \cdots, a_{n_{a}}, b_{1}, b_{2}, \cdots, b_{n_{b}}\right] \in R^{n_{a}+n_{b}} \\
\theta_{n}:=\left[c_{1}, c_{2}, \cdots, c_{n_{c}}, d_{1}, d_{2}, \cdots, d_{n_{d}}\right] \in R^{n_{c}+n_{d}}
\end{gathered}
$$

$$
\begin{gathered}
\varphi(t):=\left[\begin{array}{l}
\varphi_{s}(t) \\
\varphi_{n}(t)
\end{array}\right] \in R^{n} \\
\varphi_{s}(t):=\left[-x(t-1),-x(t-2), \cdots,-x\left(t-n_{a}\right),\right. \\
\left.u(t-1), u(t-2), \cdots, u\left(t-n_{b}\right)\right]^{T} \in R^{\left(n_{a}+n_{b}\right)} \\
\varphi_{n}(t):=\left[-w(t-1),-w(t-2), \cdots,-w\left(t-n_{c}\right),\right. \\
\left.v(t-1), v(t-2), \cdots, v\left(t-n_{d}\right)\right]^{T} \in R^{\left(n_{c}+n_{d}\right)}
\end{gathered}
$$

Define the intermediate variable,

$$
x(t):=\frac{A(z)}{B(z)} u(t)
$$

Or

$$
\begin{aligned}
x(t)= & {[1-A(z)] x(t)+B(z) u(t) } \\
= & \left(-a_{1} z^{-1}-a_{2} z^{-2}-\cdots-a_{n_{a}} z^{-n_{a}}\right) x(t) \\
& +\left(b_{1} z^{-1}+b_{2} z^{-2}+\cdots+b_{n_{b}} z^{-n_{b}}\right) u(t) \\
= & -a_{1} x(t-1)-a_{2} x(t-2)-\cdots \\
& -a_{n_{a}} x\left(t-n_{a}\right)+b_{1} u(t-1)+b_{2} u(t-2) \\
& +\cdots+b_{n_{b}} u\left(t-n_{b}\right) \\
= & \varphi_{s}^{T}(t) \theta_{s} \\
\quad w(t):=\frac{D(z)}{C(z)} v(t) & \\
\text { Or } & \\
w(t)= & {[1-C(z)] w(t)+D(z) v(t) } \\
= & \left(-c_{1} z^{-1}-c_{2} z^{-2}-\cdots-c_{n_{c}} z^{-n_{c}}\right) w(t) \\
& +\left(1+d_{1} z^{-1}+d_{2} z^{-2}+\cdots+d_{n_{d}} z^{-n_{d}}\right) v(t) \\
= & -c_{1} w(t-1)-c_{2} w(t-2)-\cdots \\
& -c_{n_{c}} w\left(t-n_{c}\right)+v(t)+d_{1} v(t-1) \\
& +d_{2} v(t-2)+\cdots+d_{n_{d}} v\left(t-n_{d}\right) \\
= & \varphi_{n}^{T}(t) \theta_{n}+v(t)
\end{aligned}
$$

Replacing (1) with (2), (3), we have

$$
\begin{aligned}
y(t) & =x(t)+w(t) \\
& =\varphi_{s}^{T}(t) \theta_{s}+\varphi_{n}^{T}(t) \theta_{n}+v(t) \\
& =\varphi^{T}(t) \theta+v(t)
\end{aligned}
$$

\section{Two-stage least squares based iterative identification algorithm}

The basic idea of two-stage least squares iterative identification algorithm is decomposing the system into two subsystems, and decomposing the parameters and information vector respectively into two parameter sub vectors and information sub vectors, and then using hierarchical identification of the interactive estimation theory and the auxiliary model to identify parameters of each subsystem. Define two intermediate variables:

$$
\begin{aligned}
& y_{1}(t):=y(t)-\varphi_{n}^{T}(t) \theta_{n} \\
& y_{2}(t):=y(t)-\varphi_{s}^{T}(t) \theta_{s}
\end{aligned}
$$


From (5) and (6), the system in (4) can be decomposed into two "fictitious" subsystems:

$$
\begin{aligned}
& y_{1}(t)=\varphi_{s}^{T}(t) \theta_{s}+v(t) \\
& y_{2}(t)=\varphi_{n}^{T}(t) \theta_{n}+v(t)
\end{aligned}
$$

Consider the data from $t=L, L \gg n$, and define the stacked output vectors $Y(L), Y_{1}(L)$ and $Y_{2}(L)$, the stacked information matrices and $\Phi_{s}(L)$ and $\Phi_{n}(L)$, and the stacked white noise vector $V(L)$ as:

$Y(L):=\left[\begin{array}{c}y(1) \\ y(2) \\ \vdots \\ y(L)\end{array}\right] \in R^{L}$

$$
V(L):=\left[\begin{array}{c}
v(1) \\
v(2) \\
\vdots \\
v(L)
\end{array}\right] \in R^{L}
$$

$Y_{1}(L):=\left[\begin{array}{c}y_{1}(1) \\ y_{1}(2) \\ \vdots \\ y_{1}(L)\end{array}\right] \in R^{L}$

$Y_{2}(L):=\left[\begin{array}{c}y_{2}(1) \\ y_{2}(2) \\ \vdots \\ y_{2}(L)\end{array}\right] \in R^{L}$

$\Phi_{s}(L):=\left[\begin{array}{c}\varphi_{s}^{T}(1) \\ \varphi_{s}^{T}(2) \\ \vdots \\ \varphi_{s}^{T}(L)\end{array}\right] \in R^{L \times\left(n_{a}+n_{b}\right)} \Phi_{n}(L):=\left[\begin{array}{c}\varphi_{n}^{T}(1) \\ \varphi_{n}^{T}(2) \\ \vdots \\ \varphi_{n}^{T}(L)\end{array}\right] \in R^{L \times\left(n_{c}+n_{d}\right)}$

Note that $Y(L), \Phi_{s}(L)$ and $\Phi_{n}(L)$ contain all the measured data $\{u(t), y(t): t=1,2, \cdots, L\}$, from (5) and (6), we have

$$
\begin{aligned}
& Y_{1}(L)=Y(L)-\Phi_{n}(L) \theta_{n} \\
& Y_{2}(L)=Y(L)-\Phi_{s}(L) \theta_{s}
\end{aligned}
$$

From (7) and (8), we have

$$
\begin{aligned}
& Y_{1}(L)=\Phi_{s}(L) \theta_{s}+V(L) \\
& Y_{2}(L)=\Phi_{n}(L) \theta_{n}+V(L)
\end{aligned}
$$

Define two quadratic criterion functions:

$$
\begin{aligned}
& J_{1}\left(\theta_{s}\right):=\left\|Y_{1}(L)-\Phi_{s} \theta_{s}\right\|^{2} \\
& J_{2}\left(\theta_{n}\right):=\left\|Y_{2}(L)-\Phi_{n} \theta_{n}\right\|^{2}
\end{aligned}
$$

For these two optimization problems, letting the partial derivatives of $J_{1}\left(\theta_{s}\right)$ and $J_{2}\left(\theta_{n}\right)$ with respect to $\theta_{s}$ and $\theta_{n}$ be zero gives:

$$
\begin{aligned}
& \frac{\partial J_{1}\left(\theta_{s}\right)}{\partial \theta_{s}}=-2 \Phi_{s}^{T}(L)\left[Y_{1}(L)-\Phi_{s}(L) \theta_{s}\right]=0 \\
& \frac{\partial J_{2}\left(\theta_{n}\right)}{\partial \theta_{n}}=-2 \Phi_{n}^{T}(L)\left[Y_{2}(L)-\Phi_{n}(L) \theta_{n}\right]=0
\end{aligned}
$$

Assume that the information vectors $\varphi_{s}(t)$ and $\varphi_{n}(t)$ are persistently exciting, that is, $\left[\Phi_{s}^{T}(L) \Phi_{S}(L)\right]$ and $\left[\Phi_{n}^{T}(L) \Phi_{n}(L)\right]$ are non-singular. From the above two equations, we can obtain the following least squares estimates of the parameter vectors $\theta_{s}$ and $\theta_{n}$ :

$$
\begin{aligned}
& \hat{\theta}_{s}=\left[\Phi_{s}^{T}(L) \Phi_{s}(L)\right]^{-1} \Phi_{s}^{T}(L) Y_{1}(L) \\
& \hat{\theta}_{n}=\left[\Phi_{n}^{T}(L) \Phi_{n}(L)\right]^{-1} \Phi_{n}^{T}(L) Y_{2}(L)
\end{aligned}
$$

Substituting (9) into (11) and (10) into (12) gives

$$
\hat{\theta}_{s}=\left[\Phi_{s}^{T}(L) \Phi_{s}(L)\right]^{-1} \Phi_{s}^{T}(L)\left[Y(L)-\Phi_{n}(L) \theta_{n}\right]
$$$$
\hat{\theta}_{n}=\left[\Phi_{n}^{T}(L) \Phi_{n}(L)\right]^{-1} \Phi_{n}^{T}(L)\left[Y(L)-\Phi_{s}(L) \theta_{s}\right]
$$

However, the right-hand sides of (13) and (14) contain the unknown parameter $\theta_{s}$ and $\theta_{n}$, and $\Phi_{s}(L)$ and $\Phi_{n}(L)$ contain the unknown variable $x(t-i), w(t-i)$ and $v(t-i)$, respectively, it is impossible to compute the estimates $\hat{\theta}_{s}$ and $\hat{\theta}_{n}$. In order to solve the difficult, the approach is based on the hierarchical identification principle. As follows: let $k=1,2,3, \cdots$ be an iteration variable, $\hat{\theta}_{k}=\left[\begin{array}{c}\hat{\theta}_{s, k} \\ \hat{\theta}_{n, k}\end{array}\right]$ be the iterative estimate of $\theta_{k}=\left[\begin{array}{l}\theta_{s, k} \\ \theta_{n, k}\end{array}\right]$ at iteration $k$, and $\hat{x}_{k-1}(t-$ $i), \hat{w}_{k-1}(t-i)$ and $\hat{v}_{k-1}(t-i)$ be the iterative estimate of $x(t-i), w(t-i)$ and $v(t-i)$ at iteration $k-1$, and define

$$
\begin{gathered}
\hat{\varphi}_{k}(t):=\left[\begin{array}{c}
\hat{\varphi}_{s, k}(t) \\
\hat{\varphi}_{n, k}(t)
\end{array}\right] \in R^{n_{a}+n_{b}+n_{c}+n_{d}} \\
\hat{\varphi}_{s, k}(t):=\left[-\hat{x}_{k-1}(t-1),-\hat{x}_{k-1}(t-2), \cdots,-\hat{x}_{k-1}\left(t-n_{a}\right),\right. \\
\left.u(t-1), u(t-2), \cdots, u\left(t-n_{b}\right)\right]^{T} \in R^{n_{a}+n_{b}} \\
\hat{\varphi}_{n, k}:=\left[-\hat{w}_{k-1}(t-1),-\hat{w}_{k-1}(t-2), \cdots,-\hat{w}_{k-1}\left(t-n_{c}\right),\right. \\
\left.\hat{v}_{k-1}(t-1), \hat{v}_{k-1}(t-2), \cdots, \hat{v}_{k-1}\left(t-n_{d}\right)\right]^{T} \in R^{n_{c}+n_{d}}
\end{gathered}
$$

Replacing $t$ with $t-i$ in (2), we have:

$$
x(t-i)=\varphi_{s}^{T}(t-i) \theta_{s}
$$

Replacing $\varphi_{s}(t-i)$ and $\theta_{s}$ with $\hat{\theta}_{s, k}(t-i)$ and $\hat{\theta}_{s, k}(t)$, the estimate $\hat{x}_{k}(t-i)$ of $x(t-i)$ can be computed by

$$
\hat{x}_{k}(t-i)=\hat{\varphi}_{s}^{T}(t-i) \hat{\theta}_{s, k}(t)
$$

From (3), we have

$$
w(t-i)=y(t-i)-x(t-i)
$$

Replacing $\hat{x}_{k}(t-i)$ with $x(t-i)$, the estimate $\hat{w}_{k}(t-i)$ of $w(t-i)$ can be computed by

$$
\begin{aligned}
\hat{w}_{k}(t-i) & =y(t-i)-\hat{x}_{k}(t-i) \\
& =y(t-i)-\varphi_{s, k}^{T}(t-i) \hat{\theta}_{s, k}(t)
\end{aligned}
$$

From (3), we have

$$
v(t-i)=w(t-i)-\varphi_{n}^{T}(t-i) \theta_{n}
$$

Replacing $w(t-i), \varphi_{n}(t-i)$ and $\theta_{n}$ with $\hat{w}_{k}(t-i), \hat{\varphi}_{n, k}(t-$ $i)$ and $\hat{\theta}_{n, k}(t)$, the estimate $\hat{v}_{k}(t-i)$ of $v(t-i)$ can be computed by

$$
\hat{v}_{k}(t-i)=\hat{w}_{k}(t-i)-\hat{\varphi}_{n, k}^{T}(t-i) \hat{\theta}_{n, k}(t)
$$

Certainly, $v(t-i)$ can be computed by

$$
v(t-i)=y(t-i)-\varphi^{T}(t) \theta
$$

Replacing $\varphi(t), \theta$ with $\hat{\varphi}_{k}(t), \hat{\theta}_{k}(t)$ the estimate $\hat{v}_{k}(t-i)$ of $v(t-i)$ can be computed by

$$
\hat{v}_{k}(t-i)=y(t-i)-\hat{\varphi}_{k}^{T}(t-i) \hat{\theta}_{k}(t) .
$$


To sum up, two-stage least squares based iterative identification algorithm as follow:

$$
\begin{aligned}
& \hat{\theta}_{s, k}=\left[\hat{\Phi}_{s, k}^{T}(L) \hat{\Phi}_{s, k}(L)\right]^{-1} \hat{\Phi}_{s, k}^{T}(L)[Y(L)- \\
& \left.\hat{\Phi}_{n, k}(L) \hat{\theta}_{n, k-1}\right] \\
& \hat{\theta}_{n, k}=\left[\hat{\Phi}_{n, k}^{T}(L) \hat{\Phi}_{n, k}(L)\right]^{-1} \hat{\Phi}_{n, k}^{T}(L)[Y(L)- \\
& \left.\hat{\Phi}_{s, k}(L) \hat{\theta}_{s, k}\right] \\
& Y(L)=[y(1), y(2), \cdots, y(L)]^{T} \\
& \hat{\Phi}_{s, k}(L)=\left[\hat{\varphi}_{s, k}(1), \hat{\varphi}_{s, k}(2), \cdots, \hat{\varphi}_{s, k}(L)\right]^{T} \\
& \hat{\Phi}_{n, k}(L)=\left[\hat{\varphi}_{n, k}(1), \hat{\varphi}_{n, k}(2), \cdots, \hat{\varphi}_{n, k}(L)\right]^{T} \\
& \left.u(t-1), u(t-2), \cdots, u\left(t-n_{b}\right)\right]^{T} \\
& \left.\hat{v}_{k-1}(t-1), \hat{v}_{k-1}(t-2), \cdots, \hat{v}_{k-1}\left(t-n_{d}\right)\right]^{T} \\
& \hat{\varphi}_{k}(t)=\left[\varphi_{s, k}^{T}(t), \varphi_{n, k}^{T}(t)\right]^{T} \\
& \hat{\theta}_{k}=\left[\hat{\theta}_{s, k}^{T}, \hat{\theta}_{n, k}^{T}\right]^{T} \\
& \hat{x}_{k}(t-i)=\hat{\varphi}_{s, k}^{T}(t-i) \hat{\theta}_{s, k} \\
& \hat{w}_{k}(t-i)=y(t-i)-\hat{\varphi}_{s, k}^{T}(t-i) \hat{\theta}_{s, k} \\
& \hat{v}_{k}(t-i)=y(t-i)-\hat{\varphi}_{k}^{T}(t-i) \hat{\theta}_{k}
\end{aligned}
$$

The flow chart of computing the parameter estimates $\hat{\theta}_{s, k}$ and $\hat{\theta}_{n, k}$ is shown in Fig.2.

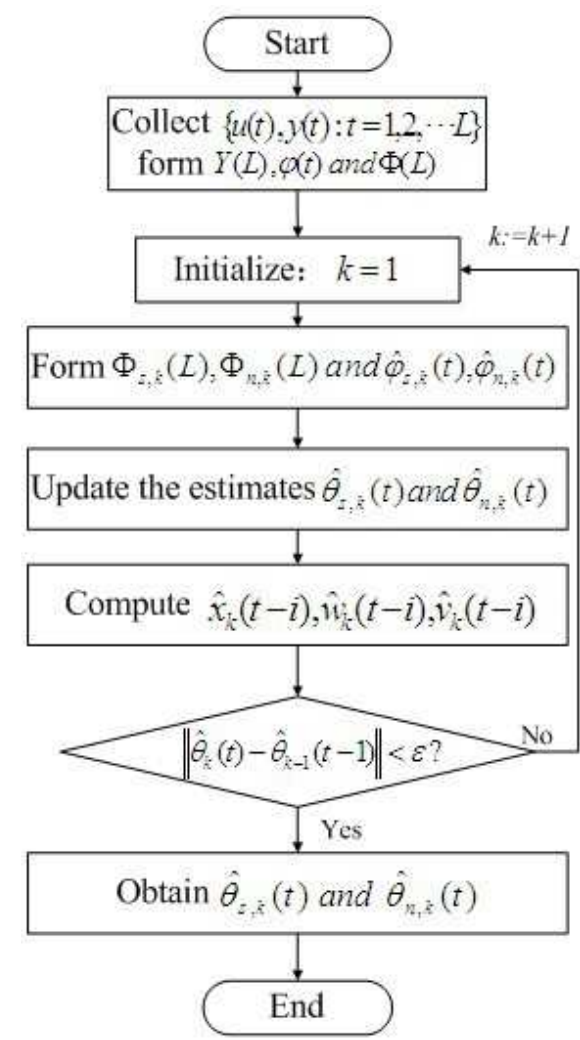

Fig. 2 The flow chart for computing the two-stage LSI parameter estimates

\section{The least squares based iterative identification algorithm}

To compare the algorithm, the least-squares iterative algorithm for Box-Jenkins model was given in this section, as follows:

$$
\begin{gathered}
\hat{\theta}_{k}=\left[\hat{\Phi}_{k}^{T}(L) \hat{\Phi}_{k}(L)\right]^{-1} \hat{\Phi}_{k}^{T}(L) Y(L) \\
Y(L)=[y(1), y(2), \cdots, y(L)]^{T} \\
\hat{\Phi}_{k}(L)=\left[\hat{\varphi}_{k}(1), \hat{\varphi}_{k}(2), \cdots, \hat{\varphi}_{k}(L)\right]^{T} \\
\hat{\varphi}_{k}(t)=\left[\begin{array}{c}
\hat{\varphi}_{s, k}(t) \\
\hat{\varphi}_{n, k}(t)
\end{array}\right] \\
\hat{\varphi}_{s, k}(t)=\left[-\hat{x}_{k-1}(t-1),-\hat{x}_{k-1}(t-2), \cdots,-\hat{x}_{k-1}\left(t-n_{a}\right),\right. \\
\left.u(t-1), u(t-2), \cdots, u\left(t-n_{b}\right)\right]^{T} \\
\hat{\varphi}_{n, k}=\left[-\hat{w}_{k-1}(t-1),-\hat{w}_{k-1}(t-2), \cdots,-\hat{w}_{k-1}\left(t-n_{c}\right),\right. \\
\left.\hat{v}_{k-1}(t-1), \hat{v}_{k-1}(t-2), \cdots, \hat{v}_{k-1}\left(t-n_{d}\right)\right]^{T} \\
\hat{\theta}_{k}=\left[\hat{\theta}_{s, k}^{T}, \hat{\theta}_{n, k}^{T}\right]^{T} \\
\hat{x}_{k}(t-i)=\hat{\varphi}_{s, k}^{T}(t-i) \hat{\theta}_{s, k} \\
\hat{w}_{k}(t-i)=y(t-i)-\varphi_{s, k}^{T}(t-i) \hat{\theta}_{s, k} \\
\hat{v}_{k}(t-i)=y(t-i)-\hat{\varphi}_{k}^{T}(t-i) \hat{\theta}_{k}
\end{gathered}
$$

\section{Example}

Consider the following simulation system of Box-Jenkins model:

$$
\begin{gathered}
y(t)=\frac{B(z)}{A(z)} u(t)+\frac{D(z)}{C(z)} v(t) \\
A(z)=1+a_{1} z^{-1}+a_{2} z^{-2}=1+1.6 z^{-1}+0.8 z^{-2} \\
B(z)=b_{1} z^{-1}+b_{2} z^{-2}=0.412 z^{-1}+0.309 z^{-2} \\
C(z)=1+c_{1} z^{-1}=1+0.8 z^{-1} \\
D(z)=1+d_{1} z^{-1}=1-0.64 z^{-1} \\
\theta_{s}=\left[a_{1}, a_{2}, b_{1}, b_{2}\right]^{T}=[1.6,0.8,0.412,0.309]^{T} \\
\theta_{n}=\left[c_{1}, d_{1}\right]^{T}=[0.8,-0.64]^{T} \\
\theta=\left[\begin{array}{c}
\theta_{s} \\
\theta_{n}
\end{array}\right]
\end{gathered}
$$

In simulation, $\{u(t)\}$ is taken as an uncorrelated persistent excitation signal sequence with zero mean and unit variance, $\{v(t)\}$ as a white noise sequence with zero mean and variance $\sigma^{2}$.

Table 1 The two-stage LSI estimates and errors ( $\mathrm{L}=3000)$

\begin{tabular}{lllllllr}
\hline$k$ & $a_{1}$ & $a_{2}$ & $b_{1}$ & $b_{2}$ & $c_{1}$ & $d_{1}$ & $\delta(\%)$ \\
\hline 1 & 0.91797 & 0.25697 & 0.41571 & 0.04388 & 0.73201 & -0.55031 & 43.21152 \\
5 & 1.49932 & 0.72201 & 0.41788 & 0.28496 & 0.80454 & -0.63611 & 6.11205 \\
10 & 1.59371 & 0.79125 & 0.41768 & 0.32386 & 0.80813 & -0.63307 & 1.03478 \\
15 & 1.56621 & 0.77580 & 0.41767 & 0.31252 & 0.80833 & -0.63235 & 2.05145 \\
20 & 1.57820 & 0.78179 & 0.41765 & 0.31749 & 0.80829 & -0.63250 & 1.51456 \\
25 & 1.57264 & 0.77918 & 0.41766 & 0.31518 & 0.80831 & -0.63241 & 1.74772 \\
26 & 1.57605 & 0.78076 & 0.41765 & 0.31660 & 0.80830 & -0.63246 & 1.60218 \\
27 & 1.57310 & 0.77939 & 0.41766 & 0.31537 & 0.80831 & -0.63242 & 1.72760 \\
True & 1.60000 & 0.80000 & 0.41200 & 0.30900 & 0.80000 & -0.64000 & 0.00000 \\
\hline
\end{tabular}




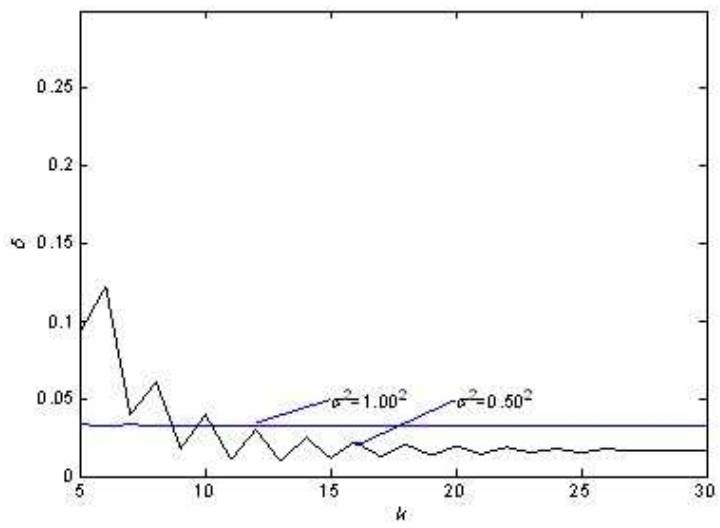

Fig. 3 the two-stage LSI parameter estimation errors $\delta$ versus $\mathrm{k}\left(\sigma^{2}=0.50^{2}\right.$ and $\left.\sigma^{2}=1.00^{2}\right)$

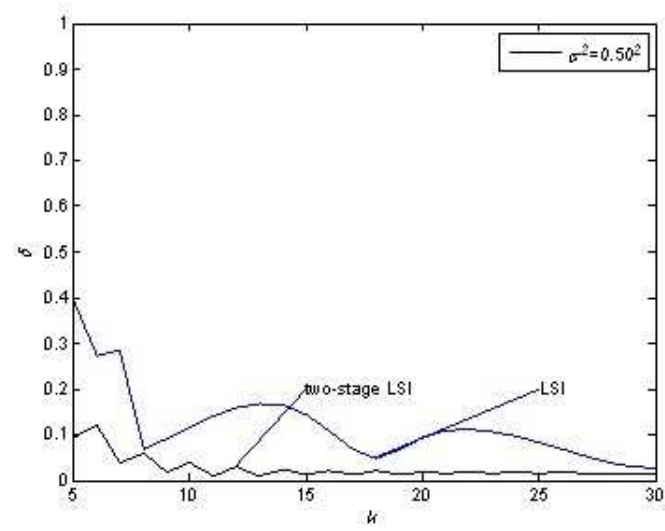

Fig. 4 the parameter estimation errors versus $k$ for the $\operatorname{algorithms}\left(\sigma^{2}=0.50^{2}\right)$

From the simulation results in Tables 1-2 and Figs.3-4, we can draw the following conclusions:

With the decrease of the noise mean variance $\sigma^{2}$, the accuracy of parameter estimation is gradually improved, compare to Fig.3;

Under the same data length and noise variance, with the increase of iteration numbers, the parameter estimation error is smaller and smaller, and the two-stage LSI is obviously superior to LSI from Fig.4

Table 2 The LSI estimates and errors $(\mathrm{L}=3000)$

\begin{tabular}{lllllllr}
\hline$k$ & $a_{1}$ & $a_{2}$ & $b_{1}$ & $b_{2}$ & $c_{1}$ & $d_{1}$ & $\delta(\%)$ \\
\hline 1 & 1.82146 & 0.93284 & 0.41597 & 0.38206 & 0.0000 & 0.0000 & 49.84331 \\
5 & 1.53821 & 0.68932 & 0.41782 & 0.25655 & 0.78749 & -0.58859 & 6.92539 \\
10 & 1.37748 & 0.63592 & 0.41822 & 0.19424 & 0.99054 & -0.67091 & 16.88002 \\
15 & 1.58786 & 0.89152 & 0.41890 & 0.28227 & 0.82818 & -0.60752 & 4.96624 \\
20 & 1.72951 & 0.91571 & 0.41825 & 0.34128 & 0.65510 & -0.66347 & 10.81336 \\
25 & 1.63762 & 0.80134 & 0.41804 & 0.30280 & 0.73493 & -0.67901 & 4.00652 \\
26 & 1.61662 & 0.78350 & 0.41804 & 0.29403 & 0.75643 & -0.67845 & 3.04469 \\
27 & 1.59680 & 0.76839 & 0.41805 & 0.28577 & 0.77707 & -0.67745 & 2.78985 \\
True & 1.60000 & 0.80000 & 0.41200 & 0.30900 & 0.80000 & -0.64000 & 0.00000 \\
\hline
\end{tabular}

\section{Conclusion}

This paper presents a two-stage LSI algorithm for BoxJenkins models by using auxiliary model identification idea and the decomposition technique. The proposed algorithm requires less computational load than the least squares based iterative algorithm. The simulation results show that the proposed algorithm has fast convergence rates and can generate highly accurate parameter estimation.

\section{Acknowledgement}

This research was supported by the National Natural Science Foundation of China (No.61263012, 61263040, 61262034), by the Postdoctoral Foundation of China (No. 2012M510593), by the Aerospace Science Foundation (No. 20120156001), by the Key Project of Chinese Ministry of Education (No. 211087), by the Natural Science Foundation of Jiangxi Province (No.20114BAB 211020, 20132BAB201025), by the Young Scientist Foundation of Jiangxi Province (No. 20122BCB23017), and by the Science and Technology Research Project of the Education Department of Jiangxi Province (No.GJJ13302).

\section{References}

[1] X. M. Xie, F. Ding, Adaptive control system, Beijing: Tsinghua university press, (2002).

[2] X. W. Chen, F. Ding, Journal of System Simulation, 20, 57585762 (2008).

[3] L. Q. Dou, Q. Q. Zong, W. J. Liu, Systems Engineering and Electronic, 31, 158-164 (2009).

[4] X. W. Chen, F. Ding, Science Technology and Engineering, 7, 5994-5992 (2007)

[5] J. H. Li, F. Ding, G. W. Yang, Mathematical and Computer Modelling, 55, 442-450 (2012).

[6] F. Ding, Control and Decision, 5, 53-56 (1990).

[7] R. J. Zhang, W. Y. Zhang, J. Wu, Journal of electrical and control, 7, 157-160 (2003).

[8] H. Z.Yang, Y.Zhang, Control Theory and Applications, 24, 215-222 (2007).

[9] H. Z.Yang, Y.Zhang, Acta Automatica Sinica, 33, 1053-1060 (2007).

[10] D. S.Chu, M. Chen, L. Li, IEEE Press, 2, 1567-1570 (2004).

[11] Y. M. Zhang, Z. Ding, H. C. Zhang, American Control Conference, 2, 1809-1813(1994).

[12] J. H. Wang, F. Ding, Science Technology and Engineering, 23, 5998-6003 (2007).

[13] J. Lu, C. X. Zhang, F. Ding, Journal of southeast university (Natural Science Edition), 38, 77-80 (2008).

[14] J. Lu, C. X. Zhang, F. Ding, Science Technology and Engineering, 8, 4683-4686 (2008).

[15] F. Ding, P. X. Liu , G. L. Liu, Digital Signal Processing, 20, 664-677 (2010).

[16] F. Ding, T. Chen..Automatica, 41, 1479-1489 (2005).

[17] H. Q. Han, L. Xie, F. Ding, X. G. Liu, Mathematical and Computer Modelling, 51, 1213-1220 (2010).

[18] Z. N. Zhang, F. Ding, X. G. Liu, Computers and Mathematics with Applications, 61, 672-682 (2011). 
[19] B. Bao, Y. Q. Xu, J. Sheng, R. F.Ding, Mathematical and Computer Modelling, 53, 1664-1669 (2011).

[20] F. Ding, Y. J. Liu, B. Bao, Journal of Systems and Control Engineering, 226, 43-55 (2012).

[21] Y. J. Lin, D. Q. Wang, F. Ding, Digital Signal Processing, 20, 1458-1467 (2010).

[22] H. H. Duan, J. Jia, R. F. Ding, Mathematical and Computer Modelling, 55, 1151-1159 (2012).

[23] G. Y. Yao, R. F. Ding, Computers and Mathematics with Applications, 63, 975-984 (2012).

[24] N. Yue, F. Z. Zhu, Y. Zhou, F. Ding, Science Technology and Engineering, 9, 1918-1920 (2009).

[25] G. Y. Yao, R. F. Ding, Computers and Mathematics with Applications, 63, 975-984 (2012).

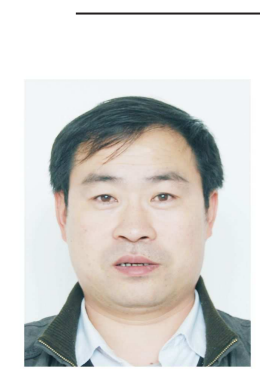

Jie Jia received his Master degree and PH.D degree at Northwestern Polytechnical University. Currently he is a professor of the School of Information Engineering at Nanchang Aeronautical University. He has published more than 30 core papers.

His research focuses on aircraft navigation guidance and control, test technology research of aeronautics and astronautics, aviation micro sensor system, the control of nonlinear system and its simulation, information modeling and simulation technology.

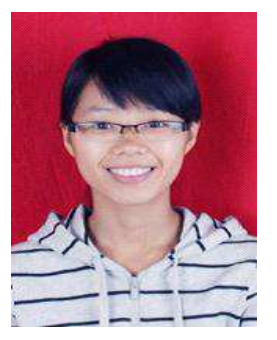

Hua Huang was born in Quannan Jiangxi on May 27, 1990 and graduated from School of Information Engneering at Nanchang Aeronautical University in July 2012. Currently she is a postgraduate and her major is Control Engineering for the School of Information Engneering at Nanchang Aeronautical University. Her research interests include intelligent control and navigation guidance and control.

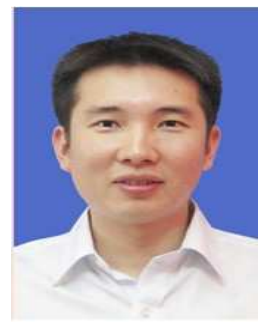

Yong Yang received his $\mathrm{Ph} . \mathrm{D}$. degree in Biomedical Engineering from $\mathrm{Xi}^{\prime}$ an Jiaotong University, China, in 2005. From 2009 to 2010, he was a postdoctoral research fellow at the Chonbuk National University, Republic of Korea. He is currently a Full Professor with the School of Information Technology, Jiangxi University of Finance and Economics, China. $\mathrm{He}$ has published more than 70 research articles in international journals and conferences. His research interests include image processing, medical image analysis, signal processing, and pattern recognition.

Ke Lv was born

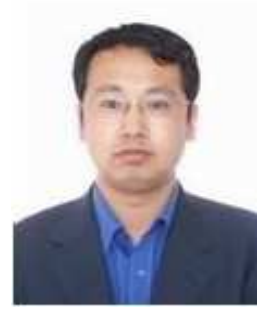
in Guyuan Ningxia on March 13, 1971, he graduated from Department of Mathematics at Ningxia University in July 1993.He received Master degree and PH.D degree from Department of Mathematics and Department of Computer science

at

Northwest University in July 1998 and July 2003, respectively. He as Postdoctoral Fellow in Institute of Automation Chinese Academy of Sciences from July 2003 to April 2005. Currently he is a professor of University of the Chinese Academy of Sciences. He research focuses on curve matching, 3D image reconstruction and computer graphics.

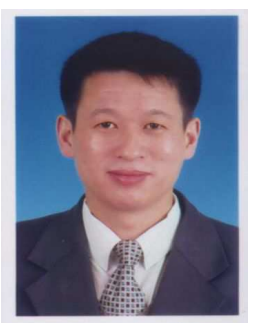

Feng Ding was born in Guangshui Hubei on 1963.He graduated from Hubei Polytechnical University in July 1984. He received Master degree and PH.D degree from Tsinghua university in July 1990 and in July 1994. He as Postdoctoral research fellow in University of Alberta from July 2003 to October 2005, in

The Hong Kong university of science and technology from March 2006 to May 2006 and Ryerson University from Janunary 2009 to October 2009. Currently he is a professor of the Internet of Things Institute in Jiangnan University. He has published more than 200 Academic papers in domestic and international professional journals and magazines. His research focuses on system modeling, system identification, process control, adaptive control. 